DOI: 10.12731/2658-6649-2019-11-4-38-55

УДК 616.12-008.331.1:616.24

\title{
ОСОБЕННОСТИ
}

\section{ПОКАЗАТЕЛЕЙ СУТОЧНОГО МОНИТОРИРОВАНИЯ АРТЕРИАЛЬНОГО ДАВЛЕНИЯ У МУЖЧИН С ХРОНИЧЕСКОЙ ОБСТРУКТИВНОЙ БОЛЕЗНЬЮ ЛЕГКИХ В УСЛОВИЯХ КОМОРБИДНОСТИ}

\section{Козлов Е.В., Яскевич Р.А., Москаленко О.Л., Кочергина К.Н.}

Цель. Изучить особенности показателей суточного мониторирования артериального давления (СМАД) у мужчин с хронической обструктивной болезнью легких (ХОБЛ) в условиях коморбидности.

Материалы и методы. Обследовано 44 пациента мужского пола с ХОБЛ в сочетании с артериальной гипертонией (АГ) и 21 пачиент с ХОБЛ без АГ. В группу сравнения вошло 32 пачиента с АГ без ХОБЛ. Протокол исследования включал: клинические, инструментальные и лабораторные методы исследования.

Результаты. Сравнительный анализ изучаемых показателей СМАД в исследуемых группах позволил выделить следующие особенности. У пациентов с ХОБЛ в условиях коморбидности выявлены более высокие среднесуточные показатели артериального давления (АД) и повымение вариабельности АД, которое может рассматриваться в качестве фактора риска неблагоприятного прогноза заболевания. Установлены статистически значимые различия по показателям величины утреннего подъема (ВУП) и скорости утреннего подъема (СУП). У пациентов ХОБЛ в условиях коморбидности преобладали патологические типь суточной кривой АД «нон-диппер» и «найт-пикер», характеризуюшиеся, прежде всего, недостаточной степенью ночного снижения АД.

Заключение. Установлено, что у пачиентов с ХОБЛ в условиях коморбидности имеются не только нарушения вариабельности АД, но и более выраженные изменения суточного профиля АД в сравнении с другими группами.

Ключевые слова: хроническая обструктивная болезнь легких; артериальная гипертония; коморбидность; суточное мониторирование артериального давления. 


\title{
FEATURES OF INDICATORS OF DAILY MONITORING OF ARTERIAL PRESSURE IN MEN WITH CHRONIC OBSTRUCTIVE PULMONARY DISEASE IN THE CONTEXT OF COMORBIDITY
}

\author{
Kozlov E.V., Yaskevich R.A., Moskalenko O.L., Kochergina K.N.
}

The purpose of the study. To study the features of indicators of daily monitoring of blood pressure in men with chronic obstructive pulmonary disease (COPD) in comorbidity.

Materials and methods. 44 male patients with COPD in combination with arterial hypertension $(A H)$ and 21 patients with COPD without AH were examined. The comparison group included 32 patients with hypertension without COPD. The study Protocol included: clinical, instrumental and laboratory methods.

Results. Comparative analysis of the studied parameters of SMAD in the studied groups allowed us to identify the following features. Patients with $C O P D$ under comorbidity conditions showed higher average daily blood pressure (BP) and increased BP variability, which can be considered as a risk factor for an unfavorable prognosis of the disease. Established statistically significant differences in terms of the magnitude of the morning rise (OPS) and the speed of the morning rise (SUP). In patients with COPD under comorbid conditions, the pathological types of the daily non-dipper and night-picker blood pressure curve prevailed, characterized primarily by an insufficient degree of night-time blood pressure reduction.

Conclusion. It was established that in patients with COPD in terms of comorbidity there are not only disturbances in the variability of blood pressure, but also more pronounced changes in the daily blood pressure profile in comparison with other groups.

Keywords: Chronic obstructive pulmonary disease; hypertension; comorbidity; daily blood pressure monitoring.

\section{Введение}

По оценкам Всемирной Организации Здравоохранения (ВО3), ХОБЛ

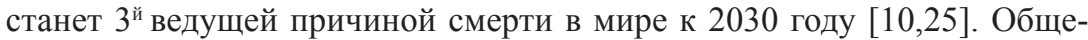
известно, что в основе ХОБЛ лежит длительно протекающий воспалительный процесс, который затрагивает все структуры легочной ткани, а 
при прогрессировании ХОБЛ приобретает системный характер, способствующий развитию атеросклеротических изменений сосудов [15]. Атеросклероз связан с повышенным риском развития инсульта, ишемической болезни сердца, заболевания периферических артерий и смертности $[9,11$, 19], однако помимо атеросклероза, вызванного воспалением, причиной сердечно-сосудистой смертности при ХОБЛ могут быть и другие факторы. Довольно часто встречается коморбидность сердечно-сосудистых заболеваний, в частности АГ и ХОБЛ $[4,12,13]$. АГ является основным фактором риска сердечно-сосудистых заболеваний и часто встречается при других респираторных заболеваниях, таких как синдром обструктивного апноэ сна (СОАС) [18]. АГ в этих случаях может быть связана с повышением симпатического тонуса [21]. Однако существует противоречивая информация о связи между АГ и ХОБЛ. В нескольких исследованиях было выявлено увеличение распространенности артериальной гипертензии среди пациентов с ХОБЛ $[22,23]$, в то время как в других исследованиях, посвященных распространенности ХОБЛ среди пациентов с артериальной гипертензией, не удалось найти этой взаимосвязи [24]. Таким образом, взгляды на генез АГ при ХОБЛ неоднозначны, но в любом случае, принимая во внимание все точки зрения не вызывает сомнения тот факт, что наличие у больных ХОБЛ сочетанной патологии в виде АГ существенно увеличивает общий сердечно-сосудистый риск [20], а проведение СМАД у этих больных актуально для своевременного выявления «скрытой» артериальной гипертензии и индивидуального подбора гипотензивной терапии $[12,14]$.

Цель: Изучить особенности показателей суточного мониторирования артериального давления у мужчин с ХОБЛ в условиях коморбидности.

\section{Материалы и методы исследования}

В исследование было включено 65 пациентов мужского пола с ХОБЛ, средний возраст 65,0 [61,5-69,5] лет. Из них пациенты с ХОБЛ в сочетании с АГ - 44 чел. (группа №2) средний возраст 66,0 [61,0-70,0] лет, пациентов с ХОБЛ без АГ 21 чел. (группа №1) средний возраст 63,0 [60,0-69,0] лет. Группу сравнения составили 32 пациента с АГ без ХОБЛ (группа № 3), средний возраст $61,0[58,0-64,5]$ лет.

Протокол исследования включал: клинические, инструментальные и лабораторные методы исследования.

Верификация диагноза ХОБЛ и оценка степени тяжести проводилась в соответствии с критериями GOLD (Global Initiative for Chronic Obstructive 
Lung Disease, update 2014) [5]. Верификацию АГ проводили согласно Рекомендациям по диагностике и лечению АГ Всероссийского научного общества кардиологов (ВНОК,2013) [17]. Всем пациентам проводилось СМАД аппаратом BPLab МнСДП-2 («Петр Телегин», Россия) в течение 24 часов. Измерения АД начинали с 9-11 часов утра. Интервалы между измерениями в дневные часы составляли 15 мин, а в ночные 30 мин. Учитывались данные, включающие в себя не менее $80 \%$ эффективных измерений.

Обработку полученных данных проводили при помощи пакета прикладных программ Statistica 6.0 (Statsoft Inc., США) [26]. Количественные значения представлялись в виде медианы (Me) [27] и межквартильного интервала в виде 25 и 75 процентилей $\left(\mathrm{Q}_{25}-\mathrm{Q}_{75}\right)$. Достоверность различий между показателями независимых выборок оценивали по непараметрическому критерию Манна-Уитни. Для сравнения показателей в нескольких группах применялся дисперсионный анализ Краскэла-Уоллиса. При сравнении групп по качественному признаку использовали критерий $\chi^{2}$. Различия считали статистически значимыми при $\mathrm{p}<0,05$.

\section{Результаты и обсуждение}

Анализируя показатели СМАД установлено, что у обследованных пациентов 2-й и 3-й группы медианы значений систолического артериального давления (САД) и диастолического артериального давления (ДАД) а также в период бодрствования и во время сна были выше в отличие от пациентов с изолированной ХОБЛ (табл. 1). При сравнении показателей АД у пациентов, имеющих АГ (группы 2 и 3 ) отмечались статистически значимые различия по показателям ДАД в ночные часы (130,5 [119,5-146,5] мм рт.ст. vs 120,0 [112,5-134,0] мм рт.ст., $\mathrm{p}=0,040)$. По среднесуточным показателям и показателям АД в дневные часы пациенты групп 2 и 3 различий не имели.

По результатам СМАД была рассчитана вариабельность САД и ДАД в период бодрствования и во время сна (табл. 1). В качестве критических значений вариабельности для САД как в дневные, так и ночные часы является показатель 15 мм рт. ст., для ДАД 14 мм рт. ст. днем и 12 мм рт. ст. ночью [16]. Вариабельность считают повышенной при превышении хотя бы одного из критических значений. Оказалось, что медианы значений вариабельности АД в период бодрствования и во время сна в 3-х группах у обследуемых лиц не выходили за пределы принятых в настоящее время нормативных показателей, однако были выше среди лиц с изолированной АГ (табл. 1). 
С целью количественной оценки эпизодов повышения АД были проанализированы показатели «нагрузки давлением», что более точно, чем средние значения АД, характеризуют нагрузку на органы-мишени. Установлено, что у мужчин с ХОБЛ в сочетании с АГ и изолированной АГ статистически значимых различий по исследуемым показателям выявлено не было ( $>0,05)$, при этом отмечена тенденция к повышению индекса площади (ИП) и индекса времени (ИВ) САД в ночные часы у пациентов в условиях коморбидности данных патологий, а у лиц с изолированной АГ имеется тенденция к более повышенным значениям по ИП и ИВ ДАД, как в период бодрствования, так и во время сна (табл. 2).

Таблиц̧а 1.

Средние значения и вариабельность АД при проведении СМАД у обследуемых больных $\left(\mathrm{Me}\left[\mathrm{Q}_{25}-\mathrm{Q}_{75}\right]\right)$

\begin{tabular}{|l|c|c|c|c|}
\hline \multicolumn{1}{|c|}{ Показатели } & $\begin{array}{c}\mathbf{1 .} \text { ХОБЛ } \\
\text { (n=21 чел.) }\end{array}$ & $\begin{array}{c}\mathbf{2} \text { ХОБЛ+АГ } \\
\text { (n=44 чел.) }\end{array}$ & $\begin{array}{c}\mathbf{3 .} \text { АГ } \\
\text { (n=32 чел.) }\end{array}$ & $p$ \\
\hline $\begin{array}{l}\text { Среднее САД (день), } \\
\text { мм рт.ст. }\end{array}$ & $\begin{array}{c}121,0 \\
{[110,0-124,0]}\end{array}$ & $\begin{array}{c}134,0 \\
{[124,5-148,5]}\end{array}$ & $\begin{array}{c}133,0 \\
{[126,0-144,5]}\end{array}$ & $\begin{array}{l}p_{1,2}=0,001 \\
p_{1,3}=0,001 \\
p_{2,3}=0,760\end{array}$ \\
\hline $\begin{array}{l}\text { Вариабельность САД } \\
\text { (день), мм рт. ст. }\end{array}$ & $\begin{array}{c}10,0 \\
{[8,0-11,0]}\end{array}$ & $\begin{array}{c}12,0 \\
{[11,0-14,0]}\end{array}$ & $\begin{array}{c}12,6 \\
{[10,35-14,8]}\end{array}$ & $\begin{array}{l}p_{1,2}=0,002 \\
p_{1,3}=0,001 \\
p_{2,3}=0,700\end{array}$ \\
\hline $\begin{array}{l}\text { Среднее САД (ночь), } \\
\text { мм рт.ст. }\end{array}$ & $\begin{array}{c}112,0 \\
{[107,0-116,0]}\end{array}$ & $\begin{array}{c}130,5 \\
{[119,5-146,5]}\end{array}$ & $\begin{array}{c}120,0 \\
{[112,5-134,0]}\end{array}$ & $\begin{array}{l}p_{1,2}=0,001 \\
p_{1,3}=0,006 \\
p_{2,3}=0,040\end{array}$ \\
\hline $\begin{array}{l}\text { Вариабельность САД } \\
\text { (ночь), мм рт. ст. }\end{array}$ & $\begin{array}{c}8,4 \\
{[8,0-10,0]}\end{array}$ & $\begin{array}{c}9,0 \\
{[7,5-13,2]}\end{array}$ & $\begin{array}{c}11,0 \\
{[8,3-12,8]}\end{array}$ & $\begin{array}{l}p_{1,2}=0,200 \\
p_{1,3}=0,020 \\
p_{2,3}=0,500\end{array}$ \\
\hline $\begin{array}{l}\text { Среднее ДАД (день), } \\
\text { мм рт.ст. }\end{array}$ & $\begin{array}{c}77,0 \\
{[70,0-85,0]}\end{array}$ & $\begin{array}{c}83,0 \\
{[77,0-91,5]}\end{array}$ & $\begin{array}{c}87,0 \\
{[79,5-93,0]}\end{array}$ & $\begin{array}{l}p_{1,2}=0,020 \\
p_{1,3}=0,001 \\
p_{2,3}=0,200\end{array}$ \\
\hline $\begin{array}{l}\text { Вариабельность ДАД } \\
\text { (день), мм рт. ст. }\end{array}$ & $\begin{array}{c}10,0 \\
{[8,0-11,0]}\end{array}$ & $\begin{array}{c}12,0 \\
{[11,0-14,0]}\end{array}$ & $\begin{array}{c}12,6 \\
{[10,35-14,8]}\end{array}$ & $\begin{array}{l}p_{1,2}=0,002 \\
p_{1,3}=0,001 \\
p_{2,3}=0,700\end{array}$ \\
\hline $\begin{array}{l}\text { Среднее ДАД (ночь), } \\
\text { мм рт.ст. }\end{array}$ & $\begin{array}{c}69,0 \\
{[66,0-76,0]}\end{array}$ & $\begin{array}{c}78,0 \\
{[71,5-87,5]}\end{array}$ & $\begin{array}{c}78,0 \\
{[66,5-87,0]}\end{array}$ & $\begin{array}{l}p_{1,2}=0,003 \\
p_{1,3}=0,002 \\
p_{2,3}=0,600\end{array}$ \\
\hline $\begin{array}{l}\text { Вариабельность ДАД } \\
\text { (ночь), мм рт. ст. }\end{array}$ & $\begin{array}{c}7,0 \\
{[6,0-8,0]}\end{array}$ & $\begin{array}{c}8,0 \\
{[5,5-10,0]}\end{array}$ & $\begin{array}{l}p_{1,2}=0,460 \\
p_{1,3}=0,005 \\
p_{2,3}=0,020\end{array}$ \\
\hline
\end{tabular}


Окончание табл. 1.

\begin{tabular}{|l|c|c|c|l|}
\hline $\begin{array}{l}\text { ЧСС (день), } \\
\text { ударов в минуту }\end{array}$ & $\begin{array}{c}75,0 \\
{[70,0-80,0]}\end{array}$ & $\begin{array}{c}80,0 \\
{[73,5-90,0]}\end{array}$ & $\begin{array}{c}72,0 \\
{[64,0-79,0]}\end{array}$ & $\begin{array}{l}p_{1,2}=0,030 \\
p_{1,3}=0,310 \\
p_{2,3}=0,0005\end{array}$ \\
\hline $\begin{array}{l}\text { Вариабельность ЧСС } \\
\text { (день), ударов } \\
\text { В минуту }\end{array}$ & $\begin{array}{c}10,0 \\
{[7,0-11,0]}\end{array}$ & $\begin{array}{c}9,0 \\
{[7,0-12,0]}\end{array}$ & $\begin{array}{c}8,9 \\
{[7,0-11,0]}\end{array}$ & $\begin{array}{l}p_{1,2}=0,980 \\
p_{1,3}=0,750 \\
p_{2,3}=0,590\end{array}$ \\
\hline $\begin{array}{l}\text { ЧСС (ночь), ударов } \\
\text { в минуту }\end{array}$ & $\begin{array}{l}66,0 \\
{[61,0-72,0]}\end{array}$ & $\begin{array}{c}69,5 \\
{[61,0-81,0]}\end{array}$ & $\begin{array}{c}59,0 \\
{[52,0-64,0]}\end{array}$ & $\begin{array}{l}p_{1,2}=0,210 \\
p_{1,3}=0,005 \\
p_{2,3}=0,001\end{array}$ \\
\hline $\begin{array}{l}\text { Вариабельность ЧСС } \\
\text { (ночь), ударов } \\
\text { в минуту }\end{array}$ & $\begin{array}{l}4,0 \\
{[3,0-7,0]}\end{array}$ & $\begin{array}{c}6,0 \\
{[5,0-9,0]}\end{array}$ & $\begin{array}{l}p_{1,2}=0,120 \\
p_{1,3}=0,990 \\
p_{2,3}=0,060\end{array}$ \\
\hline
\end{tabular}

Таблица 2.

Показатели нагрузки давлением у пациентов с АГ и ХОБЛ в сочетании с $А \Gamma\left(M e\left[Q_{25}-Q_{75}\right]\right)$

\begin{tabular}{|l|c|c|c|}
\hline \multicolumn{1}{|c|}{ Показатели } & $\begin{array}{c}\mathbf{1 . ~ Х О Б Л ~ + ~ А Г ~} \\
\text { (n=44 чел.) }\end{array}$ & $\begin{array}{c}\mathbf{2 .} \text { АГ } \\
(\mathbf{n = 3 2} \text { чел.) }\end{array}$ & $\boldsymbol{p}$ \\
\hline Индекс площади САД день & $\begin{array}{c}26,0 \\
{[4,5-115,5]}\end{array}$ & $\begin{array}{c}26,55 \\
{[9,0-103,95]}\end{array}$ & $\mathrm{p}=0,7$ \\
\hline Индекс площади САД ночь & $\begin{array}{c}62,5 \\
{[9,5-165,0]}\end{array}$ & $\begin{array}{c}22,35 \\
{[2,05-77,1]}\end{array}$ & $\mathrm{p}=0,09$ \\
\hline ИВ САД день, \% & $\begin{array}{c}23,5 \\
{[6,5-71,0]}\end{array}$ & $\begin{array}{c}24,5 \\
{[8,45-61,9]}\end{array}$ & $\mathrm{p}=0,7$ \\
\hline ИВ САД ночь, \% & 73,0 & 49,7 & $\mathrm{p}=0,1$ \\
\hline Индекс площади ДАД день & {$[31,5-100,0]$} & {$[12,2-87,7]$} & $\mathrm{p}$ \\
\hline Индекс площади ДАД ночь & {$[3,0-52,0]$} & {$[3,65-86,25]$} & $\mathrm{p}=0,3$ \\
\hline ИВ ДАД день, \% & $\begin{array}{c}35,0 \\
{[9,5-99,5]}\end{array}$ & $\begin{array}{c}49,35 \\
{[7,9-109,5]}\end{array}$ & $\mathrm{p}=0,5$ \\
\hline ИВ ДАД ночь, \% & $\begin{array}{c}16,5 \\
{[6,5-53,0]}\end{array}$ & $\begin{array}{c}34,7 \\
{[6,7-64,3]}\end{array}$ & $\mathrm{p}=0,3$ \\
\hline
\end{tabular}

Прогностически важным параметром СМАД при оценке поражений органов-мишеней является степень ночного снижения (CHC) АД, которая оценивается по суточному индексу (СИ). Как известно, неблагоприятными вариантами двухфазного ритма АД с точки зрения риска развития поражения органов-мишеней и сердечно-сосудистых осложнений являются 
типы «найтпикер» и «нондиппер». При исследовании суточного профиля АД среди обследованных групп установлено что, у мужчин с изолированной ХОБЛ доминировал тип «нон-диппер» 52,4\%, тип «найт-пикер» встречался у 19\% больных (рис. 1). У пациентов в условиях коморбидности данных патологий в отличие от групп с изолированной ХОБЛ и АГ чаще выявлялся прогностически неблагоприятный суточный профиль по САД «найт-пикер» $(27,2 \%)(\mathrm{p}>0,05)$, тип «нон-диппер» зафиксирован у $61,4 \%$ больных, что также было чаще, чем у пациентов двух других групп $(\mathrm{p}>0,05)$. У пациентов с изолированной АГ также отмечается тенденция к большей встречаемости суточного профиля по САД «нон-диппер» $50 \%$, при этом у этих больных, в сравнении с другими группами, чаще встречается физиологический тип снижения АД в ночное время «диппер», при этом достоверность наблюдается среди группы с АГ и ХОБЛ в сочетании с АГ $(37,5 \%$ и $11,4 \%$, соответственно, $\mathrm{p}<0,05)$ и реже «найт-пикер» у $12,5 \%(\mathrm{p}>0,05)$.

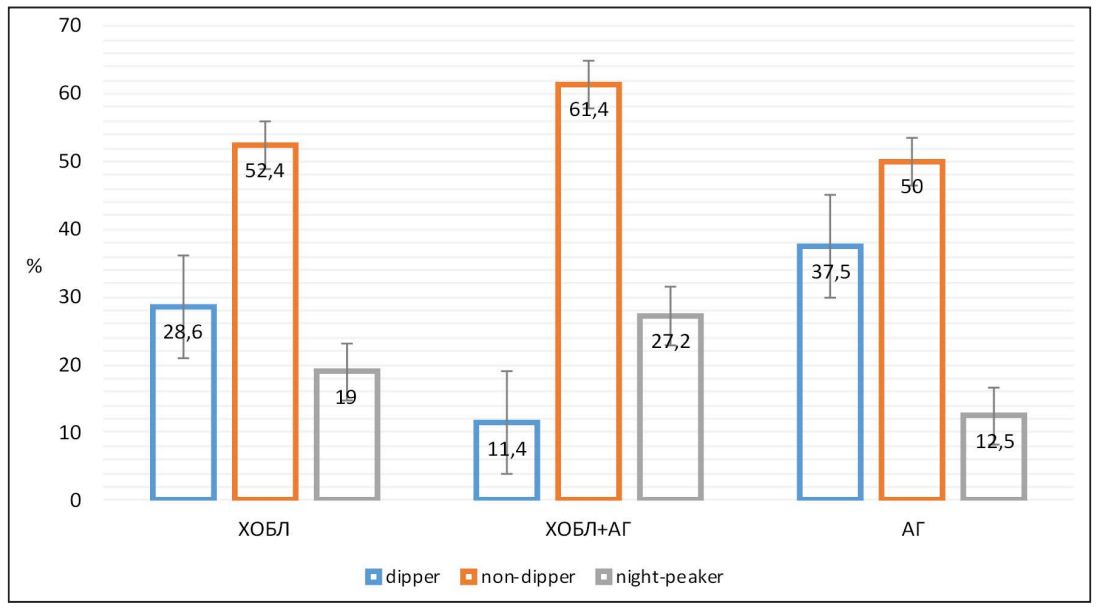

Рис. 1. Суточный профиль САД в группах исследования

Полученные результаты о преобладании среди пациентов ХОБЛ в сочетании с АГ патологических типов суточной кривой АД согласуются с данными исследования Задионченко В.С. с соавт. (2012) [7], в котором показано преобладание пациентов с повышением или отсутствием снижения АД в ночном время («найт-пикер»-31\%, «нон-диппер»- 50\%, «диппер»19\%), с данными исследования Баздырева Е.Д. с соавт. (2014), где распре- 
деление суточного профиля АД было следующим: «найт-пикер» - 34,9\%, «нон-диппер» - 51,2\%, «диппер» - 13,9\% [3]. В исследовании Адашевой Т.В. с соавт. (2011) [1], также обратило на себя внимание преобладание лиц с повышением или отсутствием снижения АД в ночном периоде у лиц с АГ и ХОБЛ («найт-пикер» - 16,5\%, «нон-диппер» - 48\%, «диппер»-35,5\%). Аксеновой Т.А. с соавт. (2013) при анализе степени ночного снижения центрального аортального давления у больных с сочетанием АГ и ХОБЛ тип «найт-пикер» отмечен в 18,5\% случаев, «нон-диппер»- в 55,5\%, «диппер» $18,5 \%$ «овер-диппер» - в 7,4\% [2].

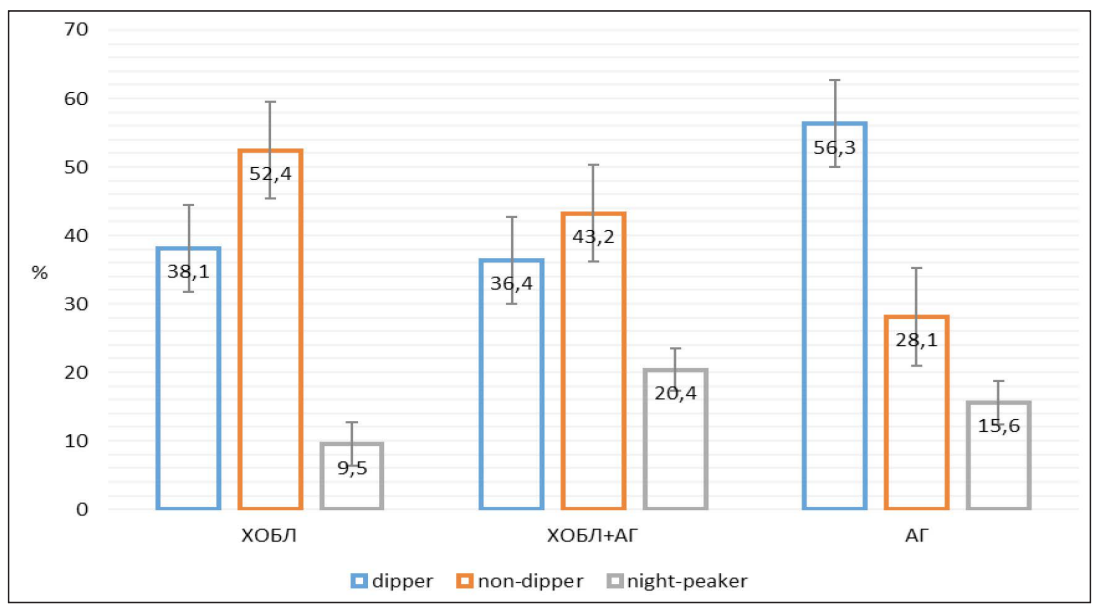

Рис. 2. Суточный профиль ДАД в группах исследования

По результатам оценки циркадной динамики суточного профиля ДАД (рис. 2) выявлено, что у пациентов 3 группы в сравнении с 1-й и 2-й группами доминирует тип «диппер» $(56,3$ \%), тип «нон-диппер» встречались с одинаковой частотой чаще у пациентов 1-й и 2-й групп. Прогностически неблагоприятный суточный профиль «найт-пикер» выявлялся чаще у пациентов в условиях коморбидности данных патологий. Суточный профиль АД «овер-диппер» среди пациентов обследуемых групп выявлен не был.

Недостаточное снижение АД в ночные часы у больных с изолированной ХОБЛ и ХОБЛ в условиях коморбидности связано, по-видимому, с тем, что во время сна у данной категории больных возникают периоды гипоксемии и гиперкапнии, приводящие к повышению активности симпатоадреналовой системы у больных ХОБЛ [8]. 
Таким образом, учитывая данные исследований СМАД у больных ХОБЛ в условиях коморбидности, пульмогенная АГ отличается преобладанием патологических типов суточной кривой АД, характеризующихся, прежде всего, недостаточной степенью ночного снижения АД.

В суточном профиле АД специального рассмотрения заслуживает его утреннее повышение. Многочисленные исследования свидетельствуют, что большинство сердечно-сосудистых катастроф приходится на утренние часы В этот период времени происходит наибольшее по сравнению с другими периодами суток число инфарктов миокарда, мозговых инсультов, злокачественных сердечных аритмий, приводящих к внезапной смерти даже у лиц с АГ без клинических проявлений коронарной болезни сердца [13].

При анализе показателей СМАД в утренние часы были получены статистически значимые различия по величине ВУП и СУП, как САД, так и ДАД у пациентов с ХОБЛ в сочетании с АГ, так и без нее, что указывает на высокую степень сердечно-сосудистого риска у данных больных (табл. 3). ВУП САД и ДАД была достоверно ниже в группе больных с изолированной ХОБЛ $(121,0$ [110,0-124,0] мм рт.ст. и 112,0 [107,0-116,0] мм рт.ст. соответственно). Выявлены статистически значимые различия по показателям СУП АД в обследованных группах, при этом показатели СУП во всех группах, включая лиц с изолированной ХОБЛ, значительно превышали нормативные показатели.

Таблииа 3 .

Показатели утренней динамика $А Д$ у обследуемых пациентов $\left(\mathrm{Me}\left[\mathrm{Q}_{25}-\mathrm{Q}_{75}\right]\right)$

\begin{tabular}{|c|c|c|c|c|}
\hline Показатели & $\begin{array}{l}\text { 1. ХОБЛ } \\
\text { (n=21 чел.) }\end{array}$ & $\begin{array}{c}\text { 2. ХОБЛ+АГ } \\
(\mathrm{n}=44 \text { чел.) }\end{array}$ & $\begin{array}{c}\text { 3. АГ } \\
(\mathrm{n}=32 \text { чел.) }\end{array}$ & $p$ \\
\hline $\begin{array}{l}\text { Величина утреннего } \\
\text { подъема САД, мм рт.ст. }\end{array}$ & $\begin{array}{c}121,0 \\
{[110,0-124,0]}\end{array}$ & $\begin{array}{c}134,0 \\
{[124,5-148,5]}\end{array}$ & $\begin{array}{c}133,0 \\
{[126,0-144,5]}\end{array}$ & $\begin{array}{l}p_{1,2}=0,4 \\
p_{1,3}=0,05 \\
p_{2,3}=0,002\end{array}$ \\
\hline $\begin{array}{l}\text { Скорость утреннего } \\
\text { подъема САД, мм рт.ст. }\end{array}$ & $\begin{array}{c}10,0 \\
{[8,0-11,0]}\end{array}$ & $\begin{array}{c}12,0 \\
{[11,0-14,0]}\end{array}$ & $\begin{array}{c}12,6 \\
{[10,35-14,8]}\end{array}$ & $\begin{array}{l}p_{1,2}=0,2 \\
p_{1,3}=0,003 \\
p_{2,3}=0,001\end{array}$ \\
\hline $\begin{array}{l}\text { Величина утреннего } \\
\text { подъема ДАД, мм рт.ст. }\end{array}$ & $\begin{array}{c}112,0 \\
{[107,0-116,0]}\end{array}$ & $\begin{array}{c}130,5 \\
{[119,5-146,5]}\end{array}$ & $\begin{array}{c}120,0 \\
{[112,5-134,0]}\end{array}$ & $\begin{array}{l}p_{1,2}=0,4 \\
p_{1,3}=0,05 \\
p_{2,3}=0,1\end{array}$ \\
\hline $\begin{array}{l}\text { Скорость утреннего } \\
\text { подъема ДАД, мм рт.ст. }\end{array}$ & $\begin{array}{c}8,4 \\
{[8,0-10,0]}\end{array}$ & $\begin{array}{c}9,0 \\
{[7,5-13,2]}\end{array}$ & $\begin{array}{c}11,0 \\
{[8,3-12,8]}\end{array}$ & $\begin{array}{l}p_{1,2}=0,7 \\
p_{1,3}=0,001 \\
p_{2,3}=0,001\end{array}$ \\
\hline
\end{tabular}




\section{Заключение}

Сравнительный анализ изучаемых показателей СМАД в исследуемых группах позволил выделить следующие особенности. У пациентов с ХОБЛ в условиях коморбидности выявлены более высокие среднесуточные показатели АД и повышение вариабельности АД, которое может рассматриваться в качестве фактора риска неблагоприятного прогноза заболевания. Установлены статистически значимые различия по показателям ВУП АД и СУП АД. У пациентов ХОБЛ в условиях коморбидности преобладали патологические типы суточной кривой АД «нон-диппер» и «найт-пикер», характеризующиеся, прежде всего, недостаточной степенью ночного снижения АД.

\section{Сиисок литературы}

1. Адашева Т.В., Задионченко В.С., Павлов С.В. и др. Эффективность и безопасность применения селективного $\beta 1$-адреноблокатора Кординорма у больных артериальной гипертонией и хронической обструктивной болезнью легких // РМЖ. 2011. Т.19 № 2. С. 1-4.

2. Аксенова Т.А., Горбунов В.В., Пархоменко Ю.В. Суточное мониторирование центрального аортального давления у больных с сочетанием гипертонической болезни и хронической обструктивной болезни легких // Клиническая медицина. 2013. Т. 91. № 7. С. 43-47.

3. Баздырев Е.Д., Герасимова Е.Б., Поликутина О.М. и др. Сердечно-сосудистые факторы риска у пациентов с хронической обструктивной болезнью легких // Клиницист. 2014. №8(1). C. 22-27. https://doi.org/10.17650/18188338-2014-1-22-27

4. Вёрткин А.Л., Ховасова Н.О. Коморбидность - новая патология. Технологии её профилактики и лечения // Архивъ внутренней медицины. 2013. (4). С. 68-72. https://doi.org/10.20514/2226-6704-2013-0-4-68-72

5. Глобальная стратегия диагностики, лечения и профилактики хронической обструктивной болезни легких (пересмотр 2014 г.) / Пер. с англ. под ред. А.С. Белевского. М.: Российское респираторное общество, 2014. 92 с.

6. Деревянных Е.В., Балашова Н.А., Яскевич Р.А., Москаленко О.Л. Частота встречаемости, структура артериальной гипертонии и комплаентность среди врачей крупной клинической больницы города Красноярска // В мире научных открытий. 2018. Т. 10. № 4. C. 223-244. https://doi.org/10.12731/ wsd-2018-4-223-244

7. Задионченко В.С., Адашева Т.В., Ли В.В. и др. Артериальная гипертензия и хроническая обструктивная болезнь легких - проблемы выбора терапии // Лечащий врач. 2012. № 7. С. 77-81. 
8. Кароли Н.А., Сергеева В.А. Суточный мониторинг артериального давления у пациентов с хронической обструктивной болезнью легких // Саратовский научно-медицинский журнал. 2009. Т. 5. № 1. С. 64-67.

9. Козлов Е.В. Структурно-функциональные изменения сердечно-сосудистой системы у пациентов, страдающих артериальной гипертонией в сочетании с хронической обструктивной болезнью легких // Сибирское медицинское обозрение. 2016. № 3 (99). С. 56-66.

10. Козлов Е.В., Деревянных Е.В., Балашова Н.А. и др. Хроническая обструктивная болезнь легких как социально-экономическое бремя взрослого населения (научный обзор) // В мире научных открытий. 2018. Т. 10. № 3. C. 180-199. https://doi.org/10.12731/wsd-2018-3-180-199.

11. Козлов Е.В., Петрова М.М., Деревянных Е.В. и др. Применение препарата «НОЛИПРЕЛ А БИ-ФОРТЕ» У мужчин пожилого возраста с хронической обструктивной болезнью легких в сочетании с артериальной гипертензией // Артериальная гипертензия. 2015. Т. 21. № 2. С. 197-205.

12. Козлов Е.В., Поликарпов Л.С., Петрова М.М., Деревянных Е.В. Системное артериальное давление в легочном стволе и вариабельность суточного мониторинга артериального давления у лиц мужского пола при коморбидности хронической обструктивной болезни легких и артериальной гипертонии // Сибирское медицинское обозрение. 2013. № 5 (83). С. 64-69.

13. Крючкова О.Н., Турна Э.Ю., Мартынюк М.А. Артериальная гипертензия у больных ХОБЛ: патогенетические особенности и суточная динамика артериального давления // Крымский терапевтический журнал. 2015. № 2 (25). С. 5-9.

14. Подъянова А.И., Костарева Р.А. Особенности показателей суточного мониторирования артериального давления у больных хронической обструктивной болезнью лёгких разной степени тяжести // Врач-аспирант. 2018. T. 89. № 4. C. 39-45.

15. Провоторов В.М., Будневский А.В., Семенкова Г.Г., Шишкина Е.С. Провоспалительные цитокины при сочетании ишемической болезни сердца и хронической обструктивной болезни легких // Клиническая медицина. 2015. №2. С. 5-9.

16. Смирнова М.И., Горбунов В.М., Курехян А.С. и др. Различные виды вариабельности артериального давления у больных артериальной гипертонией и хроническими болезнями нижних дыхательных путей // Рациональная фармакотерапия в кардиологии. 2018. №14(6). C.816-825. DOI:10.20996/18196446-2018-14-6-816-825.

17. Чазова И.Е., Чучалин А.Г., Зыков К.А., Ратова Л.Г. Диагностика и лечение пациентов с артериальной гипертонией и хронической обструктивной 
болезнью легких (Рекомендации Российского медицинского общества по артериальной гипертонии и Российского респираторного общества) // Системные гипертензии. 2013. №1. С. 5-34.

18. Barbé F, Durán-Cantolla J, Capote F, de la Peña M, Chiner E, Masa JF et al. Long-term effect of continuous positive airway pressure in hypertensive patients with sleep apnea // Am. J. Respir. Crit. Care. Med. 2010. N.181, pp. 718-726.

19. Cao J.J., Arnold A.M., Manolio T.A. et al. Association of carotid artery intimamedia thickness, plaques, and $\mathrm{C}-$ reactive protein with future cardiovascular disease and all-cause mortality: the Cardiovascular Health Study // Circulation. 2007. N.116, pp. 32-38.

20. Ghoorah K., De Soyza A., Kunadian V. Increased cardiovascular risk in patients with chronic obstructive pulmonary disease and the potential mechanisms linking the two conditions: a review // Cardiol. Rev. 2013. N.21, pp. 196-202.

21. Heindl S., Lehnert M., Criée C.P. et al. Marked sympathetic activation in patients with chronic respiratory failure // Am. J. Respir. Crit. Care. Med. 2001. N.164, pp. 597-601.

22. Mannino D.M., Thorn D., Swensen A., Holguin F. Prevalence and outcomes of diabetes, hypertension and cardiovascular disease in COPD // Eur. Respir. J. 2008. N32. P. 962-969. doi: 10.1183/09031936.00012408, 11.

23. Mapel D.W., Dedrick D., Davis K. Trends and cardiovascular comorbidities of COPD patients in the Veterans Administration Medical System, 1991-1999 // COPD. 2005. N2, pp. 35-41.

24. Müllerova H., Agustí A., Erqou S., Mapel D.W. Cardiovascular comorbidity in COPD. Systematic literature review // Chest. 2013. N144, pp. 1163-1178.

25. Ntritsos G., Franek J., Belbasis L. et al. Gender-specific estimates of COPD prevalence: a systematic review and meta-analysis. // International Journal of Chronic Obstructive Pulmonary Disease. 2018. Vol.13, pp. 1507-1514. doi:10.2147/COPD.S146390.

26. Манчук В.Т., Смирнова О.В. Использование методов нейросетевого моделирования и дискриминантного анализа для оценки состояния иммунного статуса у больных острым нелимфобластным лейкозом // Якутский медицинский журнал. 2010. № 2 (30). С. 77-79.

27. Савченко А.А., Смирнова О.В., Манчук В.Т., Москов В.И. Способ прогнозирования геморрагических осложнений после химиотерапии у больных острыми лейкозами. Патент на изобретение RUS 2324190 15.02.2006.

\section{References}

1. Adasheva T.V., Zadionchenko V.S., Pavlov S.V. i dr. EHffektivnost' i bezopasnost' primeneniya selektivnogo $\beta 1$-adrenoblokatora Kordinorma u bol'nyh 
arterial'noj gipertoniej i hronicheskoj obstruktivnoj bolezn'yu legkih [Efficacy and safety of the use of selective $\beta 1$ - adrenergic blocker Kordinorm in patients with arterial hypertension and chronic obstructive pulmonary disease]. $R M Z H$ [Russian medical journal]. 2011. V.19 № 2, pp. 1-4.

2. Aksenova T.A., Gorbunov V.V., Parhomenko Y.V. Sutochnoe monitorirovanie central'nogo aortal'nogo davleniya u bol'nyh s sochetaniem gipertonicheskoj bolezni i hronicheskoj obstruktivnoj bolezni legkih [Daily monitoring of central aortic pressure in patients with a combination of hypertension and chronic obstructive pulmonary disease]. Klinicheskaya medicina [Clinical medicine]. 2013. V. 91. № 7, pp. 43-47.

3. Bazdyrev E.D., Gerasimova E.B., Polikutina O.M. i dr. Serdechno-sosudistye faktory riska u pacientov s hronicheskoj obstruktivnoj bolezn'yu legkih [Cardiovascular risk factors in patients with chronic obstructive pulmonary disease]. Klinicist [Clinician]. 2014. №8(1), pp. 22-27. https://doi.org/10.17650/18188338-2014-1-22-27.

4. Vyortkin A.L., Hovasova N.O. Komorbidnost' - novaya patologiya. Tekhnologii eyo profilaktiki i lecheniya [Comorbidity is a new pathology. Technologies for its prevention and treatment]. Arhiv vnutrennej mediciny [Archives of internal medicine]. 2013. № (4), pp. 68-72. https://doi.org/10.20514/2226-6704-20130-4-68-72.

5. Global'naya strategiya diagnostiki, lecheniya i profilaktiki hronicheskoj obstruktivnoj bolezni legkih (peresmotr 2014 g.) [Global strategy for the diagnosis, treatment and prevention of chronic obstructive pulmonary disease (revision 2014)]. / ed. A.S. Belevsky. M.: Russian Respiratory Society. 2014. 92 p.

6. Derevyannyh E.V., Balashova N.A., Yaskevich R.A., Moskalenko O.L. CHastota vstrechaemosti, struktura arterial'noj gipertonii i komplaentnost' sredi vrachej krupnoj klinicheskoj bol'nicy goroda Krasnoyarska [The frequency of occurrence, the structure of arterial hypertension and compliance among doctors of a large clinical hospital in Krasnoyarsk]. V mire nauchnyh otkrytij [In the world of scientific discoveries]. 2018. V. 10. № 4. P. 223-244. https://doi.org/10.12731/ wsd-2018-4-223-244/

7. Zadionchenko V.S., Adasheva T.V., Li V.V. i dr. Arterial'naya gipertenziya i hronicheskaya obstruktivnaya bolezn' legkih - problemy vybora terapii [Arterial hypertension and chronic obstructive pulmonary disease - problems of choice of therapy]. Lechashchij vrach [The attending physician]. 2012. № 7, pp. 77-81.

8. Karoli N.A., Sergeeva V.A. Sutochnyj monitoring arterial'nogo davleniya u pacientov s hronicheskoj obstruktivnoj bolezn'yu legkih [Daily monitoring of blood pressure in patients with chronic obstructive pulmonary disease]. Sara- 
tovskij nauchno-medicinskij zhurnal [Saratov Journal of Medical Scientific]. 2009. V. 5. № 1, pp. 64-67.

9. Kozlov E.V. Strukturno-funkcional'nye izmeneniya serdechno-sosudistoj sistemy u pacientov, stradayushchih arterial'noj gipertoniej v sochetanii s hronicheskoj obstruktivnoj bolezn'yu legkih [Structural and functional changes of the cardiovascular system in patients suffering from arterial hypertension in combination with chronic obstructive pulmonary disease]. Sibirskoe medicinskoe obozrenie [Siberian Medical Review]. 2016. № 3 (99), pp. 56-66.

10. Kozlov E.V., Derevyannyh E.V., Balashova N.A. et al. Hronicheskaya obstruktivnaya bolezn' legkih kak social'no-ehkonomicheskoe bremya vzroslogo naseleniya (nauchnyj obzor) [Chronic obstructive pulmonary disease as a socio - economic burden of the adult population (scientific review)]. $V$ mire nauchnyh otkrytij [In the world of scientific discoveries]. 2018. V. 10. № 3, pp. 180-199. https://doi.org/10.12731/wsd-2018-3-180-199.

11. Kozlov E.V., Petrova M.M., Derevyannyh E.V. i dr. Primenenie preparata «NOLIPREL A BI-FORTE» u muzhchin pozhilogo vozrasta s hronicheskoj obstruktivnoj bolezn'yu legkih v sochetanii s arterial'noj gipertenziej [Use of the preparation "NOLIPREL A B-FORTE" in elderly men with chronic obstructive pulmonary disease in combination with arterial hypertension]. Arterial'naya gipertenziya [Arterial hypertension]. 2015. V. 21. № 2, pp. 197-205.

12. Kozlov E.V., Polikarpov L.S., Petrova M.M., Derevyannyh E.V. Sistemnoe arterial'noe davlenie v legochnom stvole i variabel'nost' sutochnogo monitoringa arterial'nogo davleniya u lic muzhskogo pola pri komorbidnosti hronicheskoj obstruktivnoj bolezni legkih i arterial'noj gipertonii [Systemic arterial pressure in the pulmonary trunk and variability of the daily monitoring of arterial pressure in males with comorbidity of chronic obstructive pulmonary disease and arterial hypertension]. Sibirskoe medicinskoe obozrenie [Siberian Medical Review]. 2013. № 5 (83), pp. 64-69.

13. Kryuchkova O.N., Turna EH.YU., Martynyuk M.A. Arterial'naya gipertenziya u bol'nyh HOBL: patogeneticheskie osobennosti i sutochnaya dinamika arterial'nogo davleniya [Hypertension in patients with COPD: pathogenetic features and daily dynamics of blood pressure]. Krymskij terapevticheskij zhurnal [Crimean therapeutic journal]. 2015. № 2 (25), pp. 5-9.

14. Pod"yanova A.I., Kostareva R.A. Osobennosti pokazatelej sutochnogo monitorirovaniya arterial'nogo davleniya u bol'nyh hronicheskoj obstruktivnoj bolezn'yu lyogkih raznoj stepeni tyazhesti [Features of indicators of daily monitoring of blood pressure in patients with chronic obstructive pulmonary disease of varying severity]. Vrach-aspirant. 2018. V. 89. № 4, pp. 39-45. 
15. Provotorov V.M., Budnevskij A.V., Semenkova G.G., SHishkina E.S. Provospalitel'nye citokiny pri sochetanii ishemicheskoj bolezni serdca i hronicheskoj obstruktivnoj bolezni legkih [Pro-inflammatory cytokines in combination with ischemic heart disease and chronic obstructive pulmonary disease]. Klinicheskaya medicina [Clinical Medicine]. 2015. №2, pp. 5-9.

16. Smirnova M.I., Gorbunov V.M., Kurekhyan A.S. i dr. Razlichnye vidy variabel'nosti arterial'nogo davleniya u bol'nyh arterial'noj gipertoniej i hronicheskimi boleznyami nizhnih dyhatel'nyh putej [Various types of blood pressure variability in patients with arterial hypertension and chronic lower respiratory tract diseases]. Racional'naya farmakoterapiya v kardiologii [Rational Pharmacotherapy in Cardiology]. 2018. №14(6). P.816-825. DOI:10.20996/1819-6446-2018-14-6-816-825.

17. Chazova I.E., Chuchalin A.G., Zykov K.A., Ratova L.G. Diagnostika i lechenie pacientov s arterial'noj gipertoniej i hronicheskoj obstruktivnoj bolezn'yu legkih (Rekomendacii Rossijskogo medicinskogo obshchestva po arterial'noj gipertonii i Rossijskogo respiratornogo obshchestva) [Diagnosis and treatment of patients with arterial hypertension and chronic obstructive pulmonary disease (Recommendations of the Russian Medical Society on Arterial Hypertension and the Russian Respiratory Society)]. Sistemnye gipertenzii [Systemic hypertension]. 2013. №1, pp. 5-34.

18. Barbé F, Durán-Cantolla J, Capote F, de la Peña M, Chiner E, Masa JF et al. Longterm effect of continuous positive airway pressure in hypertensive patients with sleep apnea. Am. J. Respir. Crit. Care. Med. 2010. N.181, pp. 718-726.

19. Cao J.J., Arnold A.M., Manolio T.A. et al. Association of carotid artery intimamedia thickness, plaques, and C-reactive protein with future cardiovascular disease and all-cause mortality: the Cardiovascular Health Study. Circulation. 2007. N.116, pp. 32-38.

20. Ghoorah K., De Soyza A., Kunadian V. Increased cardiovascular risk in patients with chronic obstructive pulmonary disease and the potential mechanisms linking the two conditions: a review. Cardiol. Rev. 2013. N.21, pp. 196-202.

21. Heindl S., Lehnert M., Criée C.P. et al. Marked sympathetic activation in patients with chronic respiratory failure. Am. J. Respir. Crit. Care. Med. 2001. N.164, pp. 597-601.

22. Mannino D.M., Thorn D., Swensen A., Holguin F. Prevalence and outcomes of diabetes, hypertension and cardiovascular disease in COPD. Eur. Respir. J. 2008. N32. S. 962-969. doi: 10.1183/09031936.00012408, 11.

23. Mapel D.W., Dedrick D., Davis K. Trends and cardiovascular comorbidities of COPD patients in the Veterans Administration Medical System, 1991-1999. COPD. 2005. N2, pp. 35-41. 
24. Müllerova H., Agustí A., Erqou S., Mapel D.W. Cardiovascular comorbidity in COPD. Systematic literature review. Chest. 2013. N144, pp. 1163-1178.

25. Ntritsos G., Franek J., Belbasis L. et al. Gender-specific estimates of COPD prevalence: a systematic review and meta-analysis. International Journal of Chronic Obstructive Pulmonary Disease. 2018. Vol.1, pp. 1507-1514. doi:10.2147/ COPD.S146390.

26. Manchuk V.T., Smirnova O.V. Ispol'zovanie metodov nejrosetevogo modelirovaniya i diskriminantnogo analiza dlya ocenki sostoyaniya immunnogo statusa u bol'nyh ostrym nelimfoblastnym lejkozom [Using methods of neural network modeling and discriminant analysis to assess the state of the immune status in patients with acute non-lymphoblastic leukemia]. Yakutskij medicinskij zhurnal [Yakut Medical Journal]. 2010. № 2 (30), pp. 77-79.

27. Savchenko A.A., Smirnova O.V., Manchuk V.T., Moskov V.I. Sposob prognozirovaniya gemorragicheskih oslozhnenij posle himioterapii u bol'nyh ostrymi lejkozami [A method for predicting hemorrhagic complications after chemotherapy in patients with acute leukemia]. Patent na izobretenie [Patent for invention] RUS 2324190 15.02.2006.

\section{ДАННЫЕ ОБ АВТОРАХ}

Козлов Евгений Вячеславович, доцент кафедры пропедевтики внутренних болезней и терапии, заведующий пульмонологическим отделением, кандидат медицинских наук

ГБОУ ВПО «КрасГМУ им. проф. Ф.В. Войно-Ясенеикого» МЗ РФ; КГБУЗ «Красноярская межрайонная клиническая больница скорой медииинской помощии имени Н.С. Карповича»

ул. Партизана Железняка, 1а, г. Красноярск, 660022, Российская Федерачия; ул. Курчатова, 17, г. Красноярск, 660062, Российская Федерачия kev-pulmonolog@mail.ru

Яскевич Роман Анатольевич, доцент кафедры пропедевтики внутренних болезней и терапии, ведущий научный сотрудник группы патологии сердечно-сосудистой системы, кандидат медицинских наук, доцент ГБОУ ВПО «КрасГМУ им. проф. Ф.В. Войно-Ясенецкого» МЗ РФ; Федеральное государственное бюджетное научное учреждение «Научно-исследовательский институт медицинских проблем Севера» ул. Партизана Железняка, 1а, г. Красноярск, 660022, Российская Федерация; ул. Партизана Железняка, 32, г. Красноярск, 660022, 
Российская Федерачия

cardio@impn.ru

Москаленко Ольга Леонидовна, старший научный сотрудник, кандидат биологических наук

Федеральное государственное бюджетное научное учреждение «Научно-исследовательский институт медицинских проблем Севера» ул. Партизана Железняка, 32, г. Красноярск, 660022, Российская Федерачия gre-ll@mail.ru

Кочергина Ксения Николаевна, студент

ГБОУ ВПО «КрасГМУ им. проф. Ф.В. Войно-Ясенеикого» МЗ РФ ул. Партизана Железняка, 1a, г. Красноярск, 660022, Российская Федераиия

ko4ergina.ksen@gmail.com

\section{DATA ABOUT THE AUTHORS}

Kozlov Evgeny Vyacheslavovich, Associate Professor at Department of Propedeutics of Internal Diseases and Therapy, Head of Pulmonology Department, Candidate of Medical Science

Krasnoyarsk State Medical University named after Professor V.F. VoinoYasenetzkiy; Krasnoyarsk Interdistrict Clinical Hospital of Emergency Medical Care. N.S. Karpovich

1a, P. Zheleznyaka St., Krasnoyarsk, 660022, Russian Federation; 17, Kurchatov St., Krasnoyarsk, 660062, Russian Federation kev-pulmonolog@mail.ru

Yaskevich Roman Anatolyevich, Associate Professor at Department of Propedeutics of Internal Diseases and Therapy, Leading Researcher of the Group Pathology of the Cardiovascular System, Candidate of Medical Science, Docent

Krasnoyarsk State Medical University named after Professor V.F. Voino-Yasenetzkiy; Scientific Research Institute of medical problems of the North

1a, P. Zheleznyaka St., Krasnoyarsk, 660022, Russian Federation; 3g, P. Zheleznyaka St., Krasnoyarsk, 660022, Russian Federation cardio@impn.ru 
Moskalenko Olga Leonidovna, Senior Researcher, Candidate of Biological Sciences

Scientific Research Institute of medical problems of the North 3g, P. Zheleznyaka St., Krasnoyarsk, 660022, Russian Federation gre-ll@mail.ru

Kochergina Ksenia Nikolaevna, student

Krasnoyarsk State Medical University named after Professor V.F. Voino-Yasenetzkiy

1a, P. Zheleznyaka St., Krasnoyarsk, 660022, Russian Federation ko4ergina.ksen@gmail.com 